\title{
Chemometric analysis of lipase-catalyzed synthesis of xylitol esters in a solvent-free
} system.

\begin{abstract}
Immobilized Candida antarctica lipase B-catalyzed esterification of xylitol and two fatty acids (capric and caproic acid) were studied in a solvent-free system. The Taguchi orthogonal array method based on three-level-four-variables with nine experiments was applied for the analysis and optimization of the reaction parameters including time, substrate molar ratio, amount of enzyme, and amount of molecular sieve. The obtained conversion was higher in the esterification of xylitol and capric acid with longer chain length. The optimum conditions derived via the Taguchi approach for the synthesis of xylitol caprate and xylitol caproate were reaction time, 29 and $18 \mathrm{~h}$; substrate molar ratio, 0.3 and 1.0; enzyme amount, 0.20 and $0.05 \mathrm{~g}$, and molecular sieve amount of $0.03 \mathrm{~g}$, respectively. The good correlation between the predicted conversions $(74.18 \%$ and $61.23 \%)$ and the actual values $(74.05 \%$ and $60.5 \%)$ shows that the model derived from the Taguchi orthogonal array can be used for optimization and better understanding of the effect of reaction parameters on the enzymatic synthesis of xylitol esters in a solvent-free system.
\end{abstract}

Keyword: Esterification; Lipase; Xylitol esters; Solvent-free system; Optimization; Taguchi method. 